on exposure to air. The factor is readily soluble in a number of alcohols and esters, slightly soluble in ethers, and relatively insoluble in aliphatic and aromatic hydrocarbons or chlorinated aliphatic hydrocarbons. Activity is strongly adsorbed on activated alumina from alcohol solutions, from which it can be partially eluted by glacial acetic acid. The factor can be chromatographed on filter paper in a variety of alcohol/water solvent systems. Good resolution was obtained in descending chromatography on Whatman filter paper No. 1, using (1:4) isopropanol/ water, where the factor has an $R_{F}=0.8$.

It is not possible at the present point in this investigation to state that antheridium-inducing factors other than that described here are not present in the culture filtrate or gametophytes of Pteridium aquilinum. However, it is clear that a single, homogeneous, relatively simple chemical substance is capable, at extremely low concentration, of directing cellular differentiation and organization. Studies on the chemical constitution of the antheridium-inducing factor described here will be continued.

This work was supported in part by a research grant (NSF Grant No. G-6144) from the National Science Foundation.

Ross B. Pringle

ULRICH NÄF

Armin C. Braun

The Rockefeller Institute,

New York 21, New York.

Feb. 1.

${ }^{1}$ Dỏpp, W., Ber. deut. bot. Gesell., 63, 139 (1950).

${ }^{2}$ Näf, U., Growth, 20, 91 (1956).

${ }^{3}$ Eames, A. J., "Morphology of Vascular Plants. Lower Groups" (McGraw-Hill, New York and London, 1936).

"Näf, U., Nature, 184, 798 (1959).

\section{Manganese Toxicity in Grevillea robusta}

Grevillea robusta A. Cunn. is commonly grown as a shade tree in East African tea plantations. Seedlings are usually raised in nurseries and transplanted to the field when about $4 \mathrm{in}$. high. Failure of the plants has been reported when they are transplanted into the red, highly acid soils characteristic of some tea areas, of which the $p \mathrm{H}$ may be as low as 4.2.

Some such seedlings, which had died within three weeks of transplanting, were sent for examination from a Kericho estate. Two specimens (on the dryweight of leaf plus stem) contained 1,940 and $>4,000$ p.p.m. of manganese respectively, and those of a healthy seedling had 880 p.p.m.

On another estate, Grevillea seeds had been nursery planted, some in a red soil $(p \mathbf{H} 4 \cdot 6)$ and others in a less-acid soil $(p H 5 \cdot 4)$. Seedlings from the former soon died (dry weight of the aerial portion of the plants about $0.05 \mathrm{gm}$.), and one specimen had a manganese content of 3,650 p.p.m., while a healthy plant from the less-acid soil had 262 p.p.m. of dry weight.

Local applications of basic slag were recommended when transplanting into acid soil. F. Haworth ${ }^{1}$ notes that in Ceylon basket plants of G. robusta and Albizia moluccana are potted in a soil limed to a $p \mathrm{H}$ value of between $6 \cdot 0$ and $7 \cdot 0$.

\section{R. Chitld}

A. N. Smith

Tea Research Institute of East Africa, Kericho, Kenya.

' Haworth, F., Tea Quarterly, 23, 114 (1952).

\section{Monoxenic Cultivation of an Enchytræid Annelid}

A CULTURE of Enchytroeus fragmentosus Bell, 1959 , was kindly sent us by Prof. A. W. Bell of Los Angeles City College in May 1959. We have been able to grow it without difficulty since that date, on rolled oats kept on moistened filter paper, and can now report success in putting it into monoxenic culture (that is, growing in the presence of a single species of associated organism-in this instance Escherichia coli).

A single specimen of $E$. fragmentosus from a xenic culture (that is, growing in the presence of a mixed microbial flora) was transferred to a Petri dish of 'Difco Nutrient Agar' previously seeded with enough $E$. coli to yield a thin continuous bacterial layer. It was allowed to migrate there and, on an average of every other day over a period of a month, was transferred to a fresh Petri dish of agar and $E$. coli. The track of the worm during each stay could be readily seen; it is thus possible to say that, because of its relatively sluggish movement under the conditions described, it seldom looped back over its path. Only after the thirteenth transfer (one month) was it found to have fragmented into several pieces. This long period without fragmentation suggested a nutritionally unfavourable state. Three short pieces were then put on to a single agar slant streaked with $E$. coli, where they grew into long worms within two weeks. A subculture was next made with one worm, followed by additional fragmentation into two.

Repeated bacteriological checks of both slants reverled only $E$. coli. We can therefore conclude that the original worm was effectively monoxenized merely by migrating for an extended period in successive thin layers of $E$. coli growing on nutrient agar.

Subsequent work has shown that nutrient agar with $E$. coli is not satisfactory by itself for indefinite subculture of $E$. fragmentosus. But grains of autoclaved rolled oats dropped on the surface of the slant provide for continued vigorous growth, and the combination so established appears satisfactory for indefinite monoxenic cultivation of the annelid.

More recently we have sometimes found it possible to axenize $E$. fragmentosus directly from xenic culture by a method based on that applied to the planarian Dugesia dorotocephala by Miller and Johnson'. In one instance, after three days of washing about 40 worms in initially sterile (sintered-glass-filtered) pond water, starting with four brief washes and continuing with two longer washes ( $24 \mathrm{hr}$. and $48 \mathrm{hr}$. respectively), we selected 25 worms and transferred them to a solution of antibiotics similar to that used by Miller and Johnson (exceptions: penicillin $G, 1,000 \mathrm{U} . / \mathrm{ml}$.; streptomycin sulphate, $100 \mathrm{\gamma} / \mathrm{ml}$. ; filipin, omitted). Worms left for 2 days thus and then washed six times in sterile pond water were found not to have been axenized. But one out of three worms put through another wash of 2 days (totalling 5 days of washing) and then 7 days in the foregoing antibiotic mixture was afterwards found to be free of bacteria.

With a number of animal organisms, both protistan and metazoan, monoxenic culture has been a preliminary to successful axenic culturo (that is, to growing in the absence of living organisms of any other species) ${ }^{3}$. Our aim with $E$, fragmentosus, as it was with rhabditid nematodes ${ }^{4}$ and is at present with rotifiers and gastrotrichs ${ }^{5}$, is to put it into axenic culture and thus make it available for nutritional 